\title{
LArPix: Demonstration of low-power 3D pixelated charge readout for liquid argon time projection chambers
}

\author{
D. A. Dwyer, ${ }^{a, 1}$ M. Garcia-Sciveres, ${ }^{a}$ D. Gnani, ${ }^{a}$ C. Grace, ${ }^{a}$ S. Kohn, ${ }^{b}$ M. Kramer, ${ }^{b}$ \\ A. Krieger, ${ }^{a}$ C. J. Lin, ${ }^{a}$ K. B. Luk, ${ }^{a, b}$ P. Madigan, ${ }^{b}$ C. Marshall, ${ }^{a}$ H. Steiner, ${ }^{a, b}$ T. Stezelberger ${ }^{a}$ \\ ${ }^{a}$ Lawrence Berkeley National Laboratory, \\ Berkeley, CA, USA \\ ${ }^{b}$ University of California - Berkeley, \\ Berkeley, CA, USA \\ E-mail: dadwyer@lbl.gov
}

\begin{abstract}
We report the demonstration of a low-power pixelated readout system designed for threedimensional ionization charge detection and digital readout of liquid argon time projection chambers (LArTPCs). Unambiguous 3D charge readout was achieved using a custom-designed system-ona-chip ASIC (LArPix) to uniquely instrument each pad in a pixelated array of charge-collection pads. The LArPix ASIC, manufactured in $180 \mathrm{~nm}$ bulk CMOS, provides 32 channels of chargesensitive amplification with self-triggered digitization and multiplexed readout at temperatures from $80 \mathrm{~K}$ to $300 \mathrm{~K}$. Using an 832-channel LArPix-based readout system with $3 \mathrm{~mm}$ spacing between pads, we demonstrated low-noise $\left(<500 \mathrm{e}^{-}\right.$RMS equivalent noise charge) and very low-power $(<100 \mu \mathrm{W} / \mathrm{channel})$ ionization signal detection and readout. The readout was used to successfully measure the three-dimensional ionization distributions of cosmic rays passing through a LArTPC, free from the ambiguities of existing projective techniques. The system design relies on standard printed circuit board manufacturing techniques, enabling scalable and low-cost production of largearea readout systems using common commercial facilities. This demonstration overcomes a critical technical obstacle for operation of LArTPCs in high-occupancy environments, such as the near detector site of the Deep Underground Neutrino Experiment (DUNE).
\end{abstract}

Keywords: Time projection chambers, Electronic detector readout concepts, Cryogenic detectors ArXiv ePrint: 1808.02969

${ }^{1}$ Corresponding author. 


\section{Contents}

1 Introduction 1

2 Readout Description 2

3 Performance Evaluation $\quad \mathbf{8}$

3.1 Leakage Current 8

3.2 Gain 8

3.3 Pedestal 8

3.4 Noise 9

3.5 Power 10

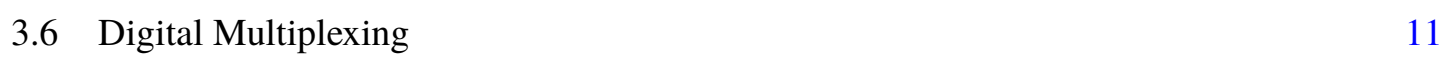

3.7 Known Issues 11

4 Demonstration of 3D Imaging of Cosmic Rays 13

5 Discussion 17

\section{Introduction}

Since its invention in 1978 [1], the time-projection chamber (TPC) has been a prominent particle tracking detector for many physics experiments [2]. Energetic particles ionize the bulk detector medium, usually gas or liquid, liberating electron-ion pairs. An externally-applied electric field drifts the electrons to a charge-collection region of the detector, also referred to as the anode. The 2D position of the charge arriving at the anode, combined with a measurement of the drift time, determines the full 3D spatial distribution of ionization within the TPC. This results in a fully-active tracking detector with homogeneous characteristics, with no need for instrumentation in the bulk volume. The ability to drift electrons in cryogenic noble liquids enables a TPC to function as both a tracking detector and a calorimeter. The ability to construct large ( $>100$ ton) liquid argon time-projection chambers (LArTPCs) at a reasonable cost makes them attractive for detecting weakly-interacting neutrinos [3-5], and was realized by the ICARUS experiment [6]. Consequently, large LArTPCs are particularly interesting to the current international program in neutrino oscillation [7-10].

The typical charge readout technique for large LArTPCs uses a stack of planes of closelyspaced parallel wires at the anode. The drifting electrons induce signals on nearby wires, and hence each plane provides an image of the charges in two dimensions: the spatial position of each wire versus drift time. Multiple wire planes can provide stereoscopic views that help estimate the location of ionization in three dimensions, but not without ambiguity when many wires receive simultaneous signals. Particle tracks or showers which are oriented nearly coplanar with the wire 
readout are particularly difficult to reconstruct. These ambiguities are exacerbated in high-intensity environments where the signals from multiple unrelated particle interactions overlap within the TPC volume, such as the proposed near-site detector of the Deep Underground Neutrino Experiment (DUNE) [10]. Recent experience with wire-based readout of large LArTPCs demonstrate the challenges not only of signal analysis, but also of noise and mechanical fragility [11-14].

Unambiguous 3D imaging of LArTPC charge signals is possible using a readout system based on a pixelated array of charged-sensitive pads. This technology is common in gaseous TPCs $[2,15,16]$, but has not until recently been considered viable for LArTPCs. Much of the difficulty stems from the substantial increase in the number of electronics channels required for pad readout compared to wire readout. Pad-based systems usually locate the front-end electronics close to the sensor plane to allow digital multiplexing to reduce the signals to a manageable number of readout channels. For such a system to function in a LArTPC, requirements beyond those for wirebased electronics must be met. In particular, these electronics must be capable of operating in liquid $\operatorname{argon}(87 \mathrm{~K})$ and must consume very little power $(<100 \mu \mathrm{W} /$ channel $)$ to avoid excessive heating of the liquid argon. Absent electronics that meet these requirements, recent demonstrations of pad readout in LArTPCs adapted existing wire-based electronics by combining the signals from tens to hundreds of pads into a single front-end electronics channel [17]. To mitigate the ambiguities from this arrangement, additional front-end channels were used to instrument complementary "regions of interest" of the anode. Comparison of the multi-pad signals with the region-of-interest signals could partially resolve ambiguities. These results highlight the importance of electronics that can provide independent readout of each pad, enabling a true 3D LArTPC.

Here we report the demonstration of a pixelated readout system that provides true $3 \mathrm{D}$ readout for LArTPCs. The specifications and design of the readout are discussed in section 2. Evaluation of the readout at both room and cryogenic temperatures is given in section 3. The demonstration of true 3D imaging of cosmic ray muon tracks in LArTPCs is presented in section 4. Concluding remarks are given in section 5 .

\section{Readout Description}

The LArPix readout system is designed to detect and acquire signals from minimum-ionizing particles (MIPs) in LArTPCs. Liquid argon has a density of $1.395 \mathrm{~g} / \mathrm{cm}^{3}$ and a boiling point of 87.3 $\mathrm{K}$ at standard atmospheric pressure. The most probable energy loss for minimum-ionizing muons in argon at this density is $1.66 \mathrm{MeV} / \mathrm{cm}$. The mean energy required to produce an electron-ion pair in liquid argon is $23.6 \mathrm{eV}[18,19]$. At a TPC drift field of $500 \mathrm{~V} / \mathrm{cm}, \sim 30 \%$ of the electrons are lost to prompt recombination with ions at MIP ionization densities [20, 21]. This results in $\sim 5000$ free electrons per mm of muon track. While additional losses to impurities (e.g. $\mathrm{O}_{2}, \mathrm{H}_{2} \mathrm{O}$ ) can further reduce signal amplitude, recent detectors have demonstrated electron lifetimes in excess of $3 \mathrm{~ms}$ which alleviates this concern [8,22]. Pad spacings in the range of $3 \mathrm{~mm}$ to $5 \mathrm{~mm}$ are of interest for large LArTPCs. At coarser spacings particle identification is hampered [23], while electron diffusion during drift reduces the advantages of finer spacings. The typical signal amplitude per pad depends on the density of pads, with pads at smaller spacings (i.e. higher densities) collecting fewer electrons per pad. The typical signal from minimum-ionizing muons at $3 \mathrm{~mm}$ spacing is roughly 15,000 electrons per pad, although the specific signal amplitudes depend on a variety of 
factors (e.g. track inclination relative to the readout, geometric overlap of the track with a given pad, electron diffusion during drift). A total system noise specification of 500 electron equivalent noise charge (ENC) corresponds to a 30:1 signal-to-noise ratio (SNR) for minimum-ionizing muon

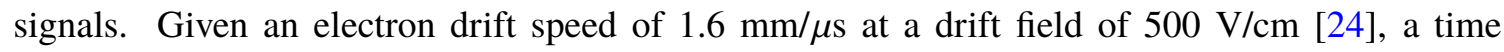
resolution of approximately $2 \mu$ s yields a spatial resolution in the drift direction comparable to the pad spacing.

Pad densities range from $40 \mathrm{k}$ to $111 \mathrm{k}$ per square meter at the pad spacings of interest, as shown in Table 1. The heat load on large LArTPCs is dominated by the heat flow through the detector cryostat walls, which is generally on the order of $10 \mathrm{~W} / \mathrm{m}^{2}$. The total area of the anodes for these LArTPCs is typically 0.5 to 1 times the inner surface area of the cryostat. If the power density of the readout is kept under $10 \mathrm{~W} / \mathrm{m}^{2}$, then the total heat load from the readout is less than the heat flux through the cryostat walls and therefore manageable using existing cooling techniques. This limits the power consumption per electronics channel to less than 90 to $250 \mu \mathrm{W}$ depending on pad density, as shown in Table 1. Detrimental effects from localized boiling of liquid argon can place additional requirements on local heat density. Argon gas bubbles within the drift field region of the LArTPC can result in electrostatic breakdown, while bubbles near the sensitive analog inputs to the electronics can produce spurious signals. Existing LArTPCs have been operated with wire-based readout electronics that have local heat densities of $\sim 0.1 \mathrm{~W} / \mathrm{cm}^{2}$, setting a general target. The observation and suppression of bubbling during operation of our pixelated readout system is discussed in section 3 .

Table 1. Pad densities and limits for average power consumption versus pad spacing. Average power limits are determined assuming a maximum power density of $10 \mathrm{~W} / \mathrm{m}^{2}$.

\begin{tabular}{crr}
\hline \hline $\begin{array}{c}\text { Pad Spacing } \\
(\mathrm{mm})\end{array}$ & $\begin{array}{r}\text { Pad Density } \\
\left(\mathrm{m}^{-2}\right)\end{array}$ & $\begin{array}{r}\text { Average Power, Upper Limit } \\
(\mu \mathrm{W} / \text { channel })\end{array}$ \\
\hline 3 & 111,111 & 90 \\
\hline 4 & 62,500 & 160 \\
\hline 5 & 40,000 & 250 \\
\hline \hline
\end{tabular}

We designed and assembled a pixelated readout system to meet these requirements. Even in high-intensity environments, the $\mathrm{O}\left(0.1 \mathrm{~cm}^{2}\right)$ area of each pad results in an expected signal rate of $\ll 1 \mathrm{~Hz}$ per pad. These low rates enable a design where much of the electronics is dormant, expending little power, until a signal occurs. The LArPix application-specific integrated circuit (ASIC) follows this approach. Each of the 32 channels on a LArPix chip functions as an independent self-triggering signal processor, responsible for amplification, digitization, and multiplexed readout. The latter stages of digitization and readout only occur when the signal on a pad exceeds a configurable threshold, resulting in a dramatic reduction of the power consumed by the digitizer and data $\mathrm{I} / \mathrm{O}$ system during the long intermissions between signals of interest.

A block diagram of the LArPix design is shown in Fig 1. The signal from each pad is input to a pulsed-reset inverting charge-sensitive amplifier (CSA) with a rise time of $45 \mathrm{~ns}$ and a gain of $25 \mathrm{mV} / \mathrm{fC}(4 \mu \mathrm{V}$ per electron). With a quiescent output of $0.55 \mathrm{~V}$ at $87 \mathrm{~K}$ and a saturation voltage just under $1.8 \mathrm{~V}$, this provides a dynamic range of $1.2 \mathrm{~V}$ or equivalently $3 \times 10^{5}$ electrons at this 


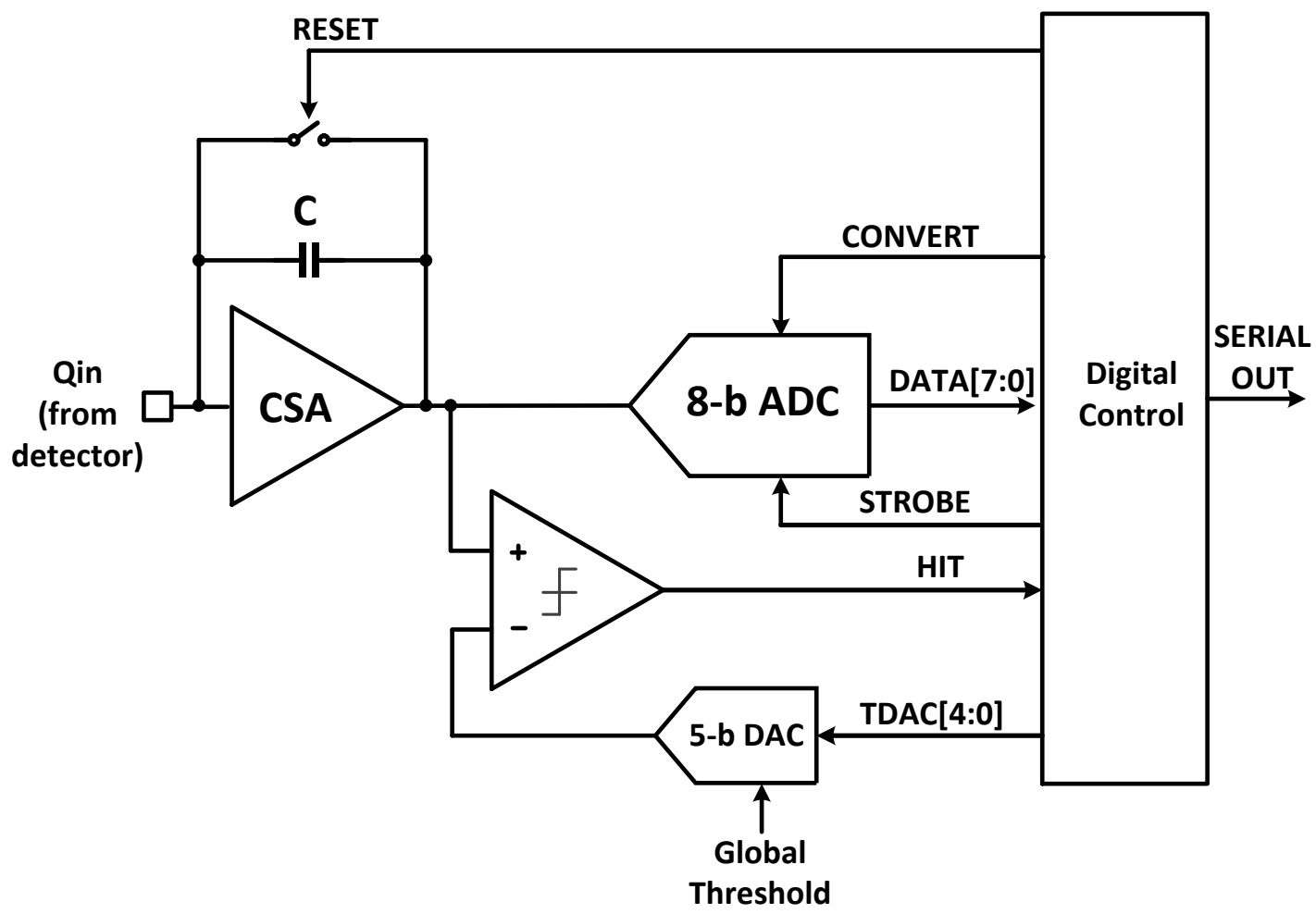

Figure 1. Block diagram of the LArPix application-specific integrated circuit (ASIC).

gain. The CSA is pulsed-reset with no continuous feedback, providing an effectively instantaneous peaking time. As electrons collect on the pad, the voltage at the output of the CSA grows until it exceeds the threshold of a self-timed discriminator with a latency of $30 \mathrm{~ns}$. A trigger from the discriminator initiates digitization of the CSA output using an 8-bit successive approximation register (SAR) analog-to-digital converter (ADC). After conversion, the digital control then resets the CSA in preparation for subsequent signals. The entire conversion and reset cycle requires 11 clock cycles, or $2.2 \mu$ s with a default $5 \mathrm{MHz}$ system clock. The ASIC is designed to support a system clock in the range of 2.5 to $20 \mathrm{MHz}$, providing a minimum time between successive ADC samples of 0.55 to $4.4 \mu \mathrm{s}$. A brief deadtime of three clock cycles occurs at the end of each triggered cycle, beginning when the CSA output is sampled by the digitizer and ending when the CSA recovers from reset.

Following each signal, the digital control writes a 54-bit digital record of the signal to an internal FIFO memory buffer where it awaits serial transmission out of the ASIC. Each hit record contains: a 2-bit record type, an 8-bit chip ID, a 7-bit channel ID, a 24-bit timestamp counter based on cycles of the system clock, an 8-bit ADC value, a 2-bit FIFO status flag, and a parity bit. The FIFO buffer on each ASIC holds up to 2048 digital records awaiting transmission out on a single data wire using a serial UART-like protocol at a rate of one bit per system clock cycle (e.g. $5 \mathrm{Mb} / \mathrm{s}$ with a $5 \mathrm{MHz}$ system clock). In a similar fashion, each ASIC is configured by passing 54-bit 
configuration digital records on a corresponding data input line. The data output line of one ASIC can be directly connected to the data input line of a subsequent ASIC; any input data records not intended for the current ASIC are passed directly to its output. This data I/O arrangement, referred to as a daisy-chain, enables communication and controls up to 256 ASICs (8192 channels) via a single pair of data input and output wires. This facilitates the operation of large readout systems ( $>10^{4}$ channels) using very few data $\mathrm{I} / \mathrm{O}$ cryostat penetrations, which is important for minimizing thermal leakage.

A variety of additional features were included in the ASIC design to allow for flexible operation and testing, with most controlled by programmable configuration registers within the ASIC. A few of the more relevant configuration options are:

1. A signal can be injected into the CSA input on any channel via an integrated pulse generator driven by an 8-bit DAC.

2. The CSA analog output from any channel can be connected to a line out for direct monitoring.

3. An 8-bit coarse threshold DAC with a range from 0 to $1.8 \mathrm{~V}$ sets a common discriminator threshold for all 32 channels per ASIC, while a fine 5-bit trim DAC accommodates relative adjustments in threshold for each channel.

4. The least-significant bit (LSB) of the trim DAC is tunable using an external bias resistor; the value used in this demonstration was $1 \mathrm{mV}$.

5. The ADC LSB can be tuned using external reference voltages, and is set to a value of $1 \mathrm{mV}$ for the measurements presented here.

6. The ADC offset is also set using an external reference voltage, accommodating the expected temperature-dependent shift of the CSA output voltage.

7. The front-end can be programmed to periodically reset without digitization, draining off any sub-threshold charge that may have accumulated on the channel.

8. A programmable channel mask inhibits self-triggering of selected channels.

9. A digital external trigger signal forces digitization and readout of channels specified via a separate programmable channel mask.

The specifications for the LArPix ASIC are summarized in Table 2. The ASICs were manufactured in $180 \mathrm{~nm}$ bulk CMOS technology at a commercial foundry. The ASIC die was $5.25 \mathrm{~mm}$ by $6.25 \mathrm{~mm}$. Only two of roughly 50 ASICs has failed to function when tested, suggesting a high yield.

A LArPix-based pixelated readout system was designed to instrument the 10-cm-diameter circular anode of a test LArTPC. A cross-section diagram of the readout assembly design is shown in figure 2. A total of 832 gold-plated copper pads spaced at $3 \mathrm{~mm}$ were etched onto one surface of a standard two-layer 1/8-inch-thick FR-4 circuit board (see left panel of figure 3), here referred to as the pixel PCB. Two pad shapes (square and triangle) and three pad sizes (large, medium, and small) were included in this design to facilitate study of their relative performance. A focusing 
Table 2. Summary of specifications for the LArPix ASIC

\begin{tabular}{lrl}
\hline \hline Specification & Value & Comment \\
\hline Analog inputs & 32 & Single-ended input \\
Gain & $4 \mu \mathrm{V} / \mathrm{e}^{-}$ & Optional high gain mode of $45 \mu{\mathrm{V} / \mathrm{e}^{-}}^{-}$ \\
Noise & $500 \mathrm{e}^{-} \mathrm{ENC}$ & \\
Power & $<100 \mu \mathrm{W} /$ channel & \\
Dynamic Range & $1.2 \mathrm{~V}$ & Corresponds to $\sim 3 \times 10^{5}$ electrons \\
ADC Resolution & $8 \mathrm{bits}$ & (See discussion in section 3.7.) \\
ADC LSB & (tunable) & Default: $2 \mathrm{mV}$ \\
Threshold range & 0 to $1.8 \mathrm{~V}$ & Tunable via global and channel trim DACs \\
Threshold resolution & $<1 \mathrm{mV}$ & Tunable via external bias resistor \\
Channel linearity & $1 \%$ & \\
Timestamp precision & 1 clock cycle & 200 ns, with a $5 \mathrm{MHz}$ system clock \\
Minimum resample time & 11 clock cycles & From $0.55 \mu$ s to $4.4 \mu$ s (2.5 to $20 \mathrm{MHz})$ \\
Operating temperature & 80 to $300 \mathrm{~K}$ & \\
FIFO event memory depth & 2048 & \\
Digital data rate & $5 \mathrm{Mb} / \mathrm{s}$ & With a $5 \mathrm{MHz}$ system clock \\
\hline \hline
\end{tabular}

grid surrounds the 416 pads on the right half of the pixel PCB. This grid is composed of a network of $0.15 \mathrm{~mm}$-wide PCB traces etched at the boundaries between these pads. Biasing this grid at voltages in the range of $-100 \mathrm{~V}$ to $-300 \mathrm{~V}$ focuses drifting electrons to increase the charge collection efficiency of these smaller pads. A grid of similar design surrounds the left-most 160 large pads, and is included to study the impact of a grounded mesh separating these pads. Vias carry the signal from each pad to the back side of this PCB, where short ( 2 to $5 \mathrm{~mm}$ ) traces route the signals from groups of 16 pads to small $1 \mathrm{~mm}$ by $5 \mathrm{~mm}$ regions for coupling to the readout electronics.

Wire bonds connect each signal trace to a unique analog input of a LArPix ASIC. A separate thin two-layer PCB, here called the data PCB, hosts the LArPix ASICs and routes the power, clock, and data input and output necessary for their operation. A data PCB designed to host four LArPix ASICs, as shown in the right panel of figure 3, was used for most of the initial characterization. Cross-talk between the digital activity on the data PCB and the pads on the pixel PCB was reduced by a shield layer consisting of a grounded copper sheet covered by polyamide to avoid electrical contact to either of the PCBs. Small $3 \mathrm{~mm}$ by $6 \mathrm{~mm}$ cavities through the data PCB and shield allow wire bond connections from each LArPix analog input directly to the signal traces on pixel PCB, as visible in figure 3 .

A custom-designed digital control system, located outside the cryogenic detector, provides power, reference voltages, a system clock, digital data input and output, and an integrated DAQ system. A Digilent Cmod A7 FPGA development board generates the system clock, and also serves as a data I/O bridge between the real-time environment of the LArPix ASICs and the DAQ computer. A miniature Raspberry Pi Zero computer running Linux (Raspbian Stretch) serves as an integrated DAQ computer and control system. A light-weight DAQ software architecture, written in Python, provides a convenient user interface for the control and operation of the readout system. 


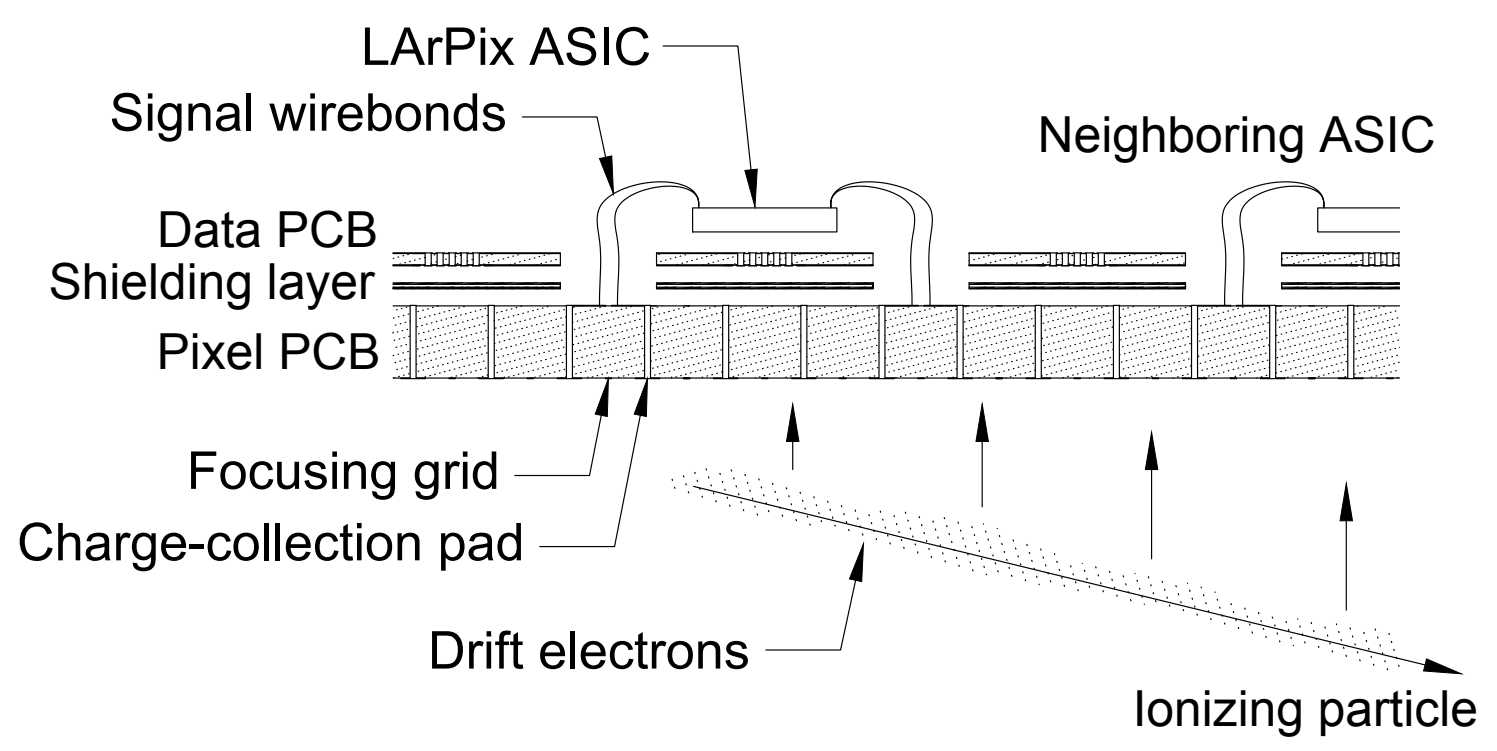

Figure 2. A cross-section diagram of the readout plane. Ionization electrons were collected on gold-plated copper pads on the Pixel PCB. The signals from each pad were transmitted via wire bonds to a unique input channel on a 32-channel LArPix ASIC. The ASIC amplified, digitized, and multiplexed the digital signals out of the system. The Data PCB provided power as well as data input and output routing for the ASIC. The shielding layer reduced the cross-talk from the Data PCB digital activity to the Pixel PCB.
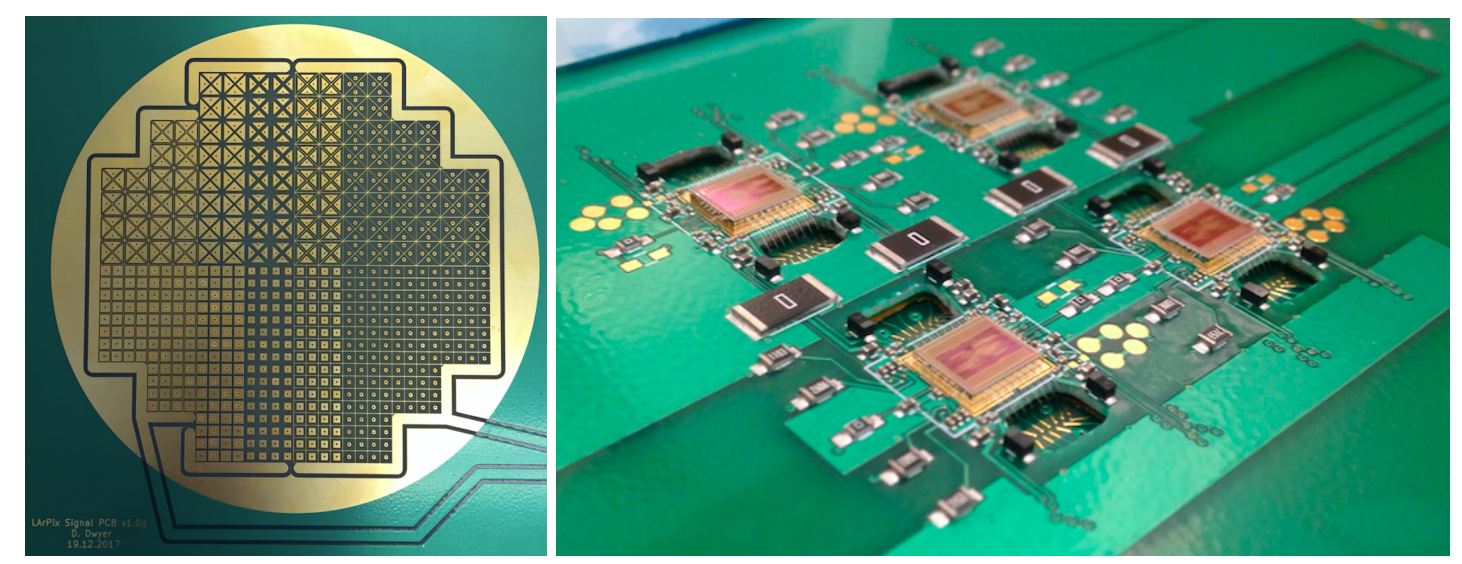

Figure 3. Left: Photograph of the TPC-facing side of a pixelated readout system. A total of 832 pads are etched on a standard two-layer circuit board. Ten different pad configurations are included in order to assess their relative performance. Right: Photograph of the back side of the readout assembly. Four LArPix ASICs are mounted on a two-layer data PCB responsible for routing system power and digital communication. For this readout system only 128 of the pads are instrumented, each wire bonded to a unique analog input of the ASICs through oblong cavities cut in the data PCB.

Communication with the DAQ computer occurs via standard 802.11 WiFi techniques, facilitating electrical isolation of the entire TPC readout system from the external environment. 


\section{Performance Evaluation}

Initial assessment of the LArPix ASICs was performed using a 128-channel (four-chip) LArPix readout system. Measurements of basic communication, gain, noise, and power were made both at room temperature and immersed in a liquid nitrogen $(\mathrm{LN})$ bath $(77 \mathrm{~K})$. Additional measurements were performed with the LArPix ASIC analog inputs left unconnected from the charge-collection pads, thereby assessing the intrinsic characteristics of the chip. No issues with the basic communication with or configuration of the ASICs were encountered under any of these conditions. Reading and writing of all of the internal configuration registers of the ASIC functioned as designed. The readout system showed no problems following multiple thermal cycles between room temperature and immersion in either LN or LAr. The details of specific measurements are discussed below.

\subsection{Leakage Current}

Using the analog monitoring feature of the LArPix ASIC, the CSA output voltage from each channel was observed to increase linearly with time. At room temperature, this increase was consistent with a small leakage current of $\sim 80 \mathrm{fA}$, or roughly 500 electrons per millisecond, into the analog input of each channel. The rising CSA output voltage would eventually exceed the discriminator threshold, resulting in self-triggering and reset at rates of a few $\mathrm{Hz}$ per channel. Enabling the periodic reset feature at rates of $3 \mathrm{kHz}$ to $10 \mathrm{kHz}$ was sufficient to drain away this slowly-accumulating charge with minimal residual bias to the CSA output voltage level. When immersed in LN, the leakage decreased to approximately $80 \mathrm{aA}\left(500 \mathrm{e}^{-} / \mathrm{s}\right)$. After bonding the pads to the ASIC channel inputs, the leakage increased by an amount which varied amongst the channels. A thorough cleaning of the pads using isopropyl alcohol was sufficient to suppress this leakage current, which was attributed to contamination on the surface of the pixel PCB.

\subsection{Gain}

The built-in pulser was used to evaluate the gain of the CSA. Pulses of increasing amplitude, as programmed using the 8-bit pulser DAC, were injected into each channel and examined using the analog monitor feature of the LArPix ASIC. This same procedure was then repeated, but with the self-trigger enabled. The resulting ADC values (calibrated to $\mathrm{mV}$ ) versus pulser DAC value are shown in figure 4. All 32 channels of this ASIC showed the expected relationship of $4 \mu \mathrm{V}$ per electron, given the approximately 1500 electrons per DAC count of the internal pulser. The precision of the internal pulser was not sufficient to assess the gain linearity at the 1\%-level given by the design specification; a precise assessment is still pending. No significant change in the gain was observed when the system was immersed in LN.

\subsection{Pedestal}

The mean DC pedestal voltage at the output of the CSA was approximately $350 \mathrm{mV}$, as measured using the analog monitoring feature of the LArPix ASIC. This mean pedestal voltage was consistent with the simulation of the LArPix front-end. The pedestal voltages were also confirmed by extrapolation of injected pulses to zero amplitude (i.e. the y-intercepts of the lines in figure 4), as well as from the distribution of ADC values obtained via forced digitization when no signal was present on the channels (see left panel of figure 5). The average pedestal voltage shifted from 


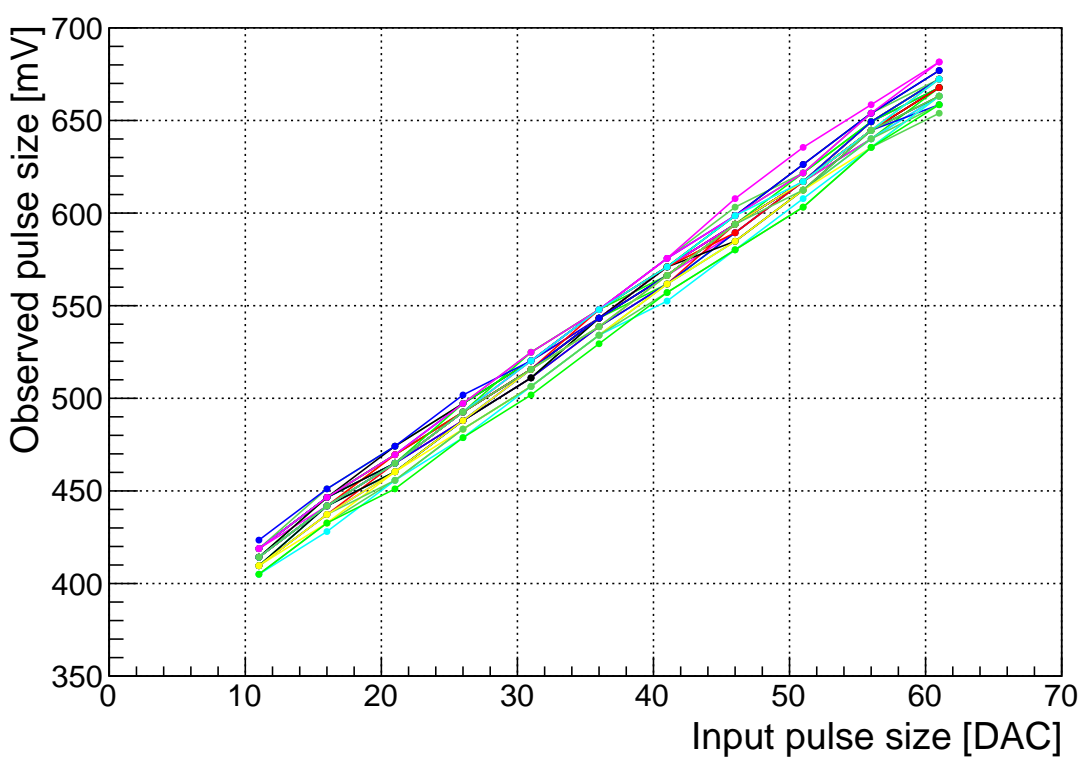

Figure 4. Plot of the self-triggered ADC value versus internal pulser DAC value for the 32 channels of one LArPix ASIC. The slope of each line provides a preliminary assessment of the CSA gain, consistent with the design specification of $4 \mu \mathrm{V}$ per electron. The digital ADC value has been converted to voltage based on an initial calibration of the ADC scale.

roughly $350 \mathrm{mV}$ at room temperature to $550 \mathrm{mV}$ in liquid nitrogen, which was also consistent with the expected temperature dependence of the front-end. The spread between the channels with the lowest and highest pedestal voltages was $30 \mathrm{mV}$ at room temperature, and increased to $50 \mathrm{mV}$ in liquid nitrogen. This channel-to-channel variation exceeded the expectations based on ASIC simulation, and was outside the default range for relative adjustment of channel thresholds (i.e. 5-bit trim DAC with a $1 \mathrm{mV}$ LSB). The trim DAC LSB is tunable through modification of external bias circuits on the Data PCB, so the LSB will be increased in future versions. The impact of insufficient trim range on channel thresholds during LArTPC operation will be discussed further in section 4.

\subsection{Noise}

The noise performance of the system was evaluated using forced digitization of each channel when no signal was present. The RMS of the ADC distribution for each channel provided a combined assessment of the noise from both the CSA as well as the digitizer. At room temperature, the 128 channels from four LArPix ASICs showed an average RMS of $1.5 \mathrm{mV}$, as displayed in the right panel of figure 5. A $1.5 \mathrm{mV}$ RMS implies an equivalent noise charge of roughly $375 \mathrm{e}^{-}$. When immersed in LN, the noise decreased to roughly $1.1 \mathrm{mV}\left(275 \mathrm{e}^{-} \mathrm{ENC}\right)$. This noise level is consistent with the design specifications shown in Table 2, and suggests a potential signal-to-noise ratio of 55:1 for minimum-ionizing muon tracks. 

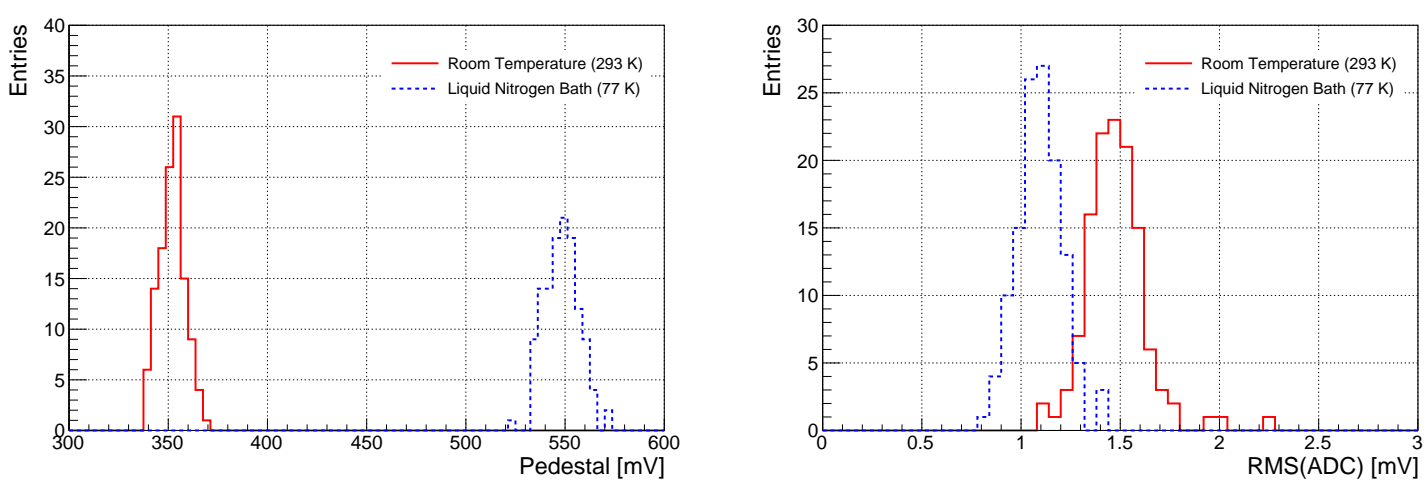

Figure 5. Left: Histograms of the pedestal voltages for 128 LArPix channels at room temperature (solid red line) and when immersed in liquid nitrogen (dashed blue line). The mean voltage is $350 \mathrm{mV}$ at room temperature. This increases to $550 \mathrm{mV}$ when the system is immersed in liquid nitrogen, which is consistent with the expected temperature dependence of the analog front-end. The spread between the channels with the lowest and highest pedestal voltages is $30 \mathrm{mV}$ at room temperature, and increases to $50 \mathrm{mV}$ in liquid nitrogen. Right: Histograms of the noise voltage of 128 LArPix channels at room temperature (solid red line) and when immersed in liquid nitrogen (dashed blue line). An average noise of $1.5 \mathrm{mV}$ (375 $\left.\mathrm{e}^{-} \mathrm{ENC}\right)$ is observed at room temperature, and decreases to $1.1 \mathrm{mV}\left(275 \mathrm{e}^{-} \mathrm{ENC}\right)$ in a liquid nitrogen bath.

\subsection{Power}

Three separate supply voltages were used to power the readout system. The analog supply voltage (VDDA), with an operating range from $+1.0 \mathrm{~V}$ to $+1.8 \mathrm{~V}$, drove the analog portion of the ASIC (CSA and part of the digitizer) and also served as a source of bias currents. The digital core supply voltage (VDDD) powered the digital functions of the ASIC (digital core and part of the digitizer), and had an operating range from approximately $+1 \mathrm{~V}$ to $+1.8 \mathrm{~V}$. The digital I/O supply voltage (VDDIO) drove the CMOS data I/O to and from the ASIC, as well as the ASIC electrostatic discharge (ESD) ring. By default VDDIO was $+3.3 \mathrm{~V}$ but had an operating range down to $+1.5 \mathrm{~V}$. Given the low-power design of the readout, we were able to power the system using three standard AAA batteries. These batteries were used in combination with three low-dropout (LDO) linear regulators (Analog Devices LT3080) and three potentiometers (Bourns 3296) to provide convenient tunable low-noise power supplies for VDDA, VDDD, and VDDIO.

Using three separate supply voltages allowed us to independently measure and tune the power consumption of each functional component of the ASIC. We measured the power consumption by placing a digital voltage meter, in current measurement mode, in series with each of the three supply voltages: VDDA, VDDD, VDDIO. Table 3 summarizes the results of these measurements when operating at room temperature and at a clock operating frequency of $5 \mathrm{MHz}$. Using an initial configuration of $+1.5 \mathrm{~V},+1.8 \mathrm{~V}$, and $+3.3 \mathrm{~V}$ for VDDA, VDDD, and VDDIO respectively, we measured an average power of $294 \mu \mathrm{W}$ per channel. We found stable operation of the readout system at VDDD values down to $+1.1 \mathrm{~V}$; at lower voltages bit errors began to occur in the data generated by the system. The digital I/O functioned correctly down to a VDDIO value of $+2.0 \mathrm{~V}$; below this value our control electronics failed to stably register the data returned by the readout system. It is possible that the system was still functional at lower VDDIO supply voltages, but we 
were unable to record and verify this data. With these lower digital supply voltages, we found an average power consumption of $62 \mu \mathrm{W}$ per channel for this 128-channel system. The power use was shared roughly equally across the analog, digital core, and digital I/O functions.

Table 3. Average power consumption per channel for a 128-channel readout system at a clock operating frequency of $5 \mathrm{MHz}$. Values are given for two configurations: an initial default configuration of the supply voltages, and a configuration tuned for low-power operation.

\begin{tabular}{ccrrr}
\hline \hline Configuration & Source & $\begin{array}{r}\text { Voltage } \\
(\mathrm{V})\end{array}$ & $\begin{array}{r}\text { Current } \\
(\mathrm{mA})\end{array}$ & $\begin{array}{r}\text { Average Power } \\
(\mu \mathrm{W} / \text { channel })\end{array}$ \\
\hline \multirow{4}{*}{ Default } & VDDA & 1.5 & 2.0 & 24 \\
& VDDD & 1.8 & 3.6 & 51 \\
& VDDIO & 3.3 & 8.5 & 219 \\
& & & & Total: 294 \\
\hline \multirow{4}{*}{ Low-power } & VDDA & 1.5 & 2.0 & 24 \\
& VDDD & 1.1 & 2.1 & 18 \\
& VDDIO & 2.0 & 1.3 & 20 \\
& & & & Total: 62 \\
\hline \hline
\end{tabular}

We also examined the variation of the power consumption with readout activity level. We lowered the threshold of one channel to the point that the data transmission through the four chip daisy chain was saturated, reaching a rate of roughly $10 \mathrm{kHz}$. This resulted in a $\sim 20 \%$ increase in total system power consumption. In a more extreme test, we lowered the thresholds on all channels such that the digital cores and internal FIFOs of all four chips were saturated. For this extreme case, the total power increased by a factor of 4 . In the expected regime of operation, with trigger rates of less than $1 \mathrm{~Hz}$ per channel, the power increased by only a few percent. Power consumption did not significantly increase when charge-collection pads were connected to the ASIC inputs, nor when the system was immersed in LN.

\subsection{Digital Multiplexing}

We exercised the digital multiplexing features of the LArPix ASIC throughout the testing program. The four chips of the readout system were operated as a single daisy chain, with all configuration and control of the system requiring only one data input and one data output line. By modification of jumpers on the data PCB, we also exercised the data I/O in configurations of 1,2, and 3 ASICs in a chain. An 896-channel daisy chain was exercised following the design and production of the 28-chip PCB shown in figure 6. This scalable layout was used to instrument all 832 pads of a pixel PCB (e.g. left panel of figure 3). In general, the daisy chain functionality performed as designed and no obstacle was found that would prevent scaling the digital multiplexing to the maximum allowed by the LArPix design: 8192 channels per pair of data I/O lines.

\subsection{Known Issues}

The following issues were identified during the development of this readout system. The default values for the ASIC configuration registers were such that the self-triggering was active with a low 


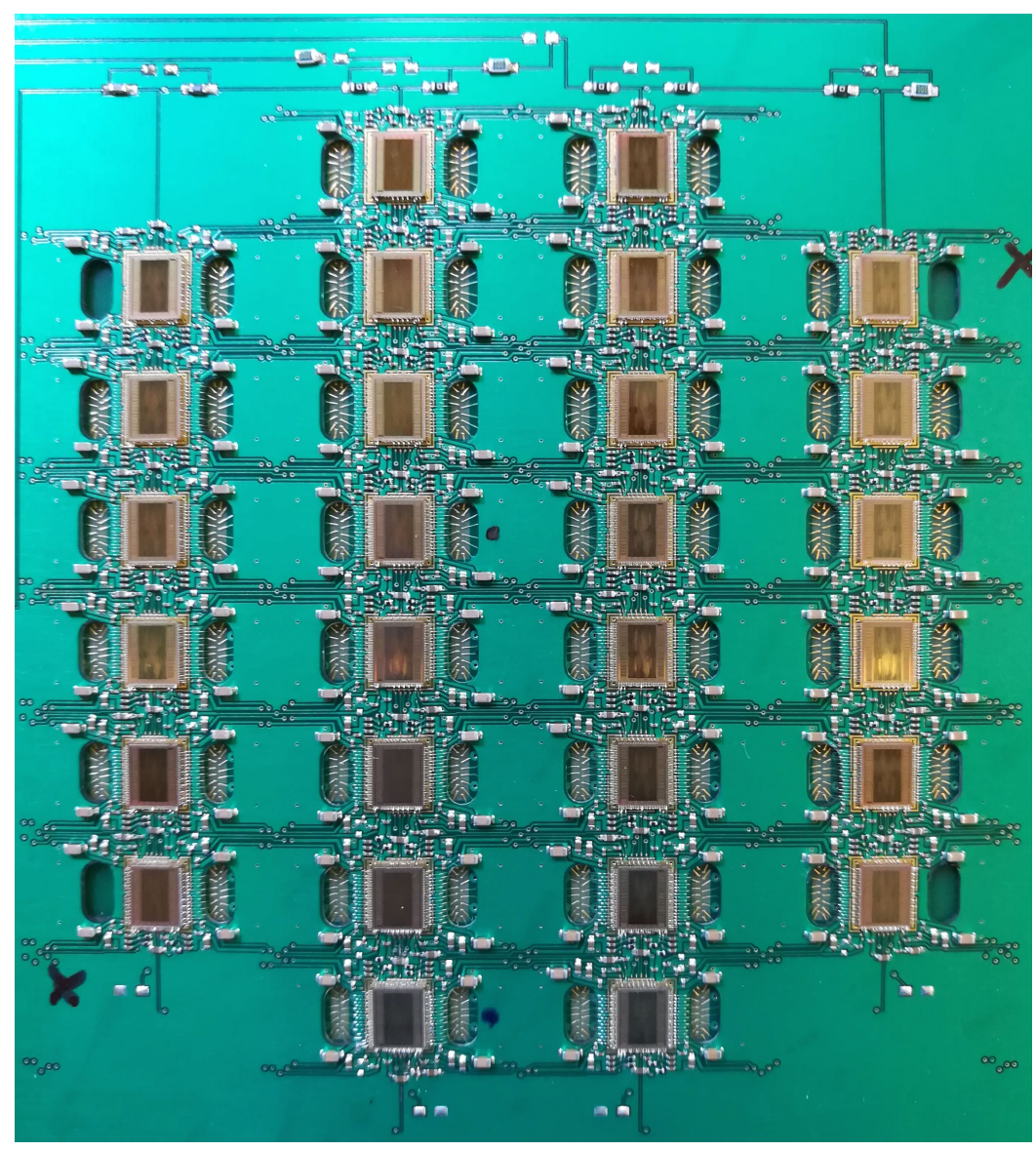

Figure 6. Photograph of the digital side of an 896-channel (28-chip) readout assembly. This larger system demonstrated scalability of the pixelated readout design, and was used to fully instrument and operate a 10-cm-diameter LArTPC.

threshold when the system was initially powered. This caused saturation of the data output and an initial increase in power consumption, but was easily resolved by prompt reconfiguration of the thresholds of all channels. The initial version of the ASIC included a 6-bit SAR ADC design, which was later enhanced to 8 bits. During testing, the ADC bits of lowest and highest significance were found to have fixed values, thus providing only the original 6-bit SAR performance. An error in the design was promptly identified via simulation, and the design was revised to provide full 8-bit performance for the next version of this ASIC. The timestamps for simultaneous externally-triggered hit records were found to be offset by 3 clock cycles. This offset resulted from a relative time delay when writing multiple simultaneous hit records to the FIFO. Synchronizing the timestamp with the trigger, instead of with the hit record entry to the FIFO, would resolve this minor issue. None of these issues presented a serious technical obstacle to operation and testing of the system, but their resolution must await production of a revised LArPix ASIC. 


\section{Demonstration of 3D Imaging of Cosmic Rays}

A small 10-cm-diameter by 10-cm-long LArTPC was used for an initial demonstration of MIP track detection using the described pixelated readout system. This TPC, shown in figure 7, was designed and built by collaborators at the University of Bern. We instrumented this TPC first with a 4-chip (128-channel) LArPix system and subsequently with a 28-chip (832-channel) system. We installed this TPC in an existing 20-liter high-purity LAr system which had been used for development and testing of liquid argon purity monitors [25]. We enhanced this system with a custom-designed capacitive liquid argon level sensor to monitor the LAr level during TPC operation, a muon telescope to provide a tagged sample of cosmic rays passing through the TPC, a resistive heater to facilitate the return to room temperature after operation, and two resistive temperature sensors for monitoring the cryostat during filling and purging as well as for monitoring the readout system temperature during operation. We arranged the system such that the cryostat lid served as the star ground point for all components internal to the cryostat, including the LArPix charge readout system. We also installed low-pass RC filters at the cathode high voltage and bias grid voltage feedthroughs on the cryostat lid. With this configuration, the noise level was found to be roughly $1.5 \mathrm{mV}$ at room temperature and $1.1 \mathrm{mV}$ in liquid argon, consistent with that observed in stand-alone tests.
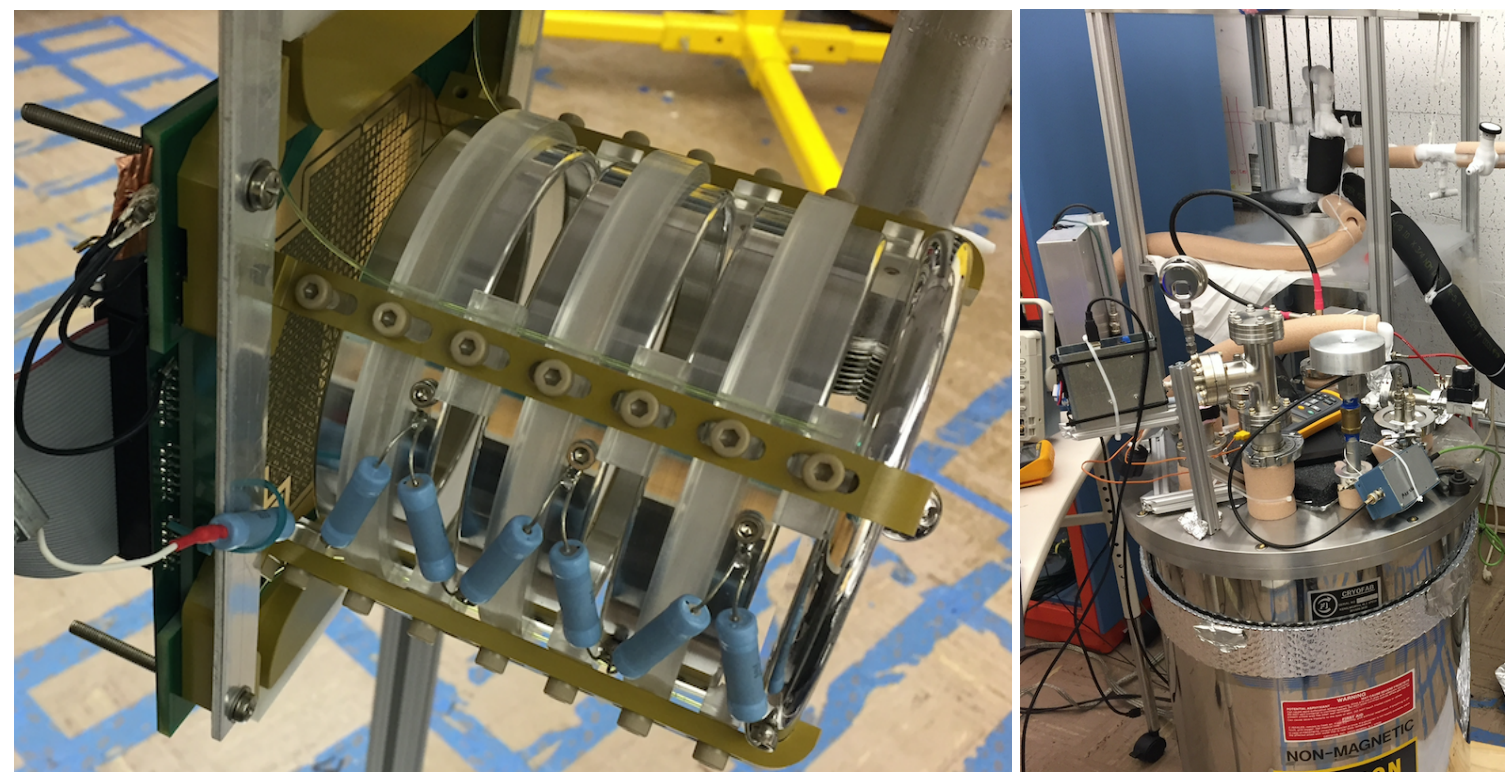

Figure 7. Left: Photograph of the 10-cm-drift TPC with a 3D readout system installed. Right: Photograph of the high-purity liquid argon system during operation. The TPC is installed in the cryostat (foreground). A single-pass of research-grade liquid argon through the LAr purifier (background) is sufficient to achieve electron lifetimes in excess of $100 \mu \mathrm{s}$.

The apparatus was filled with high-purity liquid argon using a single-pass molecular sieve and activated copper purifier to remove $\mathrm{O}_{2}$ and $\mathrm{H}_{2} \mathrm{O}$. The TPC cathode was brought to $-2 \mathrm{kV}$ using a Canberra 3002D high-voltage supply, providing a drift field of $200 \mathrm{~V} / \mathrm{cm}$ to the TPC, while the readout anode operated at ground. A separate supply (Ortec 710) was used to bias the focusing grid at $-200 \mathrm{~V}$, ensuring efficient electron collection by the smaller-sized pads. The thresholds of 
each channel were adjusted so that the hit rates from noise were less than $1 \mathrm{~Hz}$ per channel, and self-triggered data was collected.

Upon examination of this self-triggered data, the signals from cosmic ray muons were easily identified as bursts of hit records arriving within $\sim 100 \mu$ s of each other. Figure 8 shows two 3D images of cosmic ray raw data, the left panel taken with the 128-channel system and the right panel with the 832-channel system. Each large colored point in this figure represents one hit record, where the color provides a rough estimate of the charge (with blue to green approximately covering the range of $10 \mathrm{k}$ to $20 \mathrm{k}$ electrons). Each small gray point represents the position of a single pad from the pixel PCB shown in figure 3. In this display, the sensor face of the readout system is shown in a tilted perspective and each hit is displaced from the face using the relative offset of each hit timestamp. A scale of $1 \mathrm{~mm} / \mu$ s was used for this offset, consistent with the expected electron drift velocity in liquid argon at an applied field of $200 \mathrm{~V} / \mathrm{cm}$.

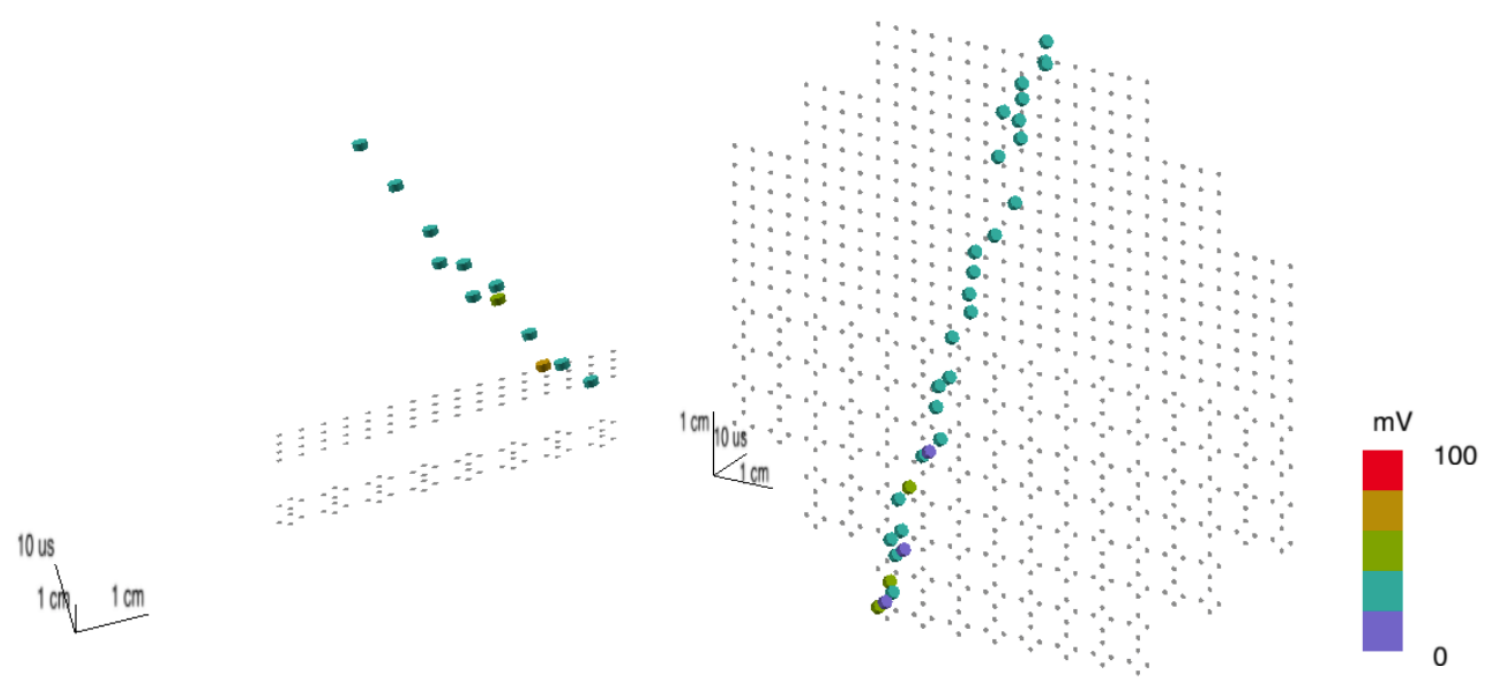

Figure 8. Left: $3 \mathrm{D}$ image of a cosmic ray muon which passed at a steep angle relative to the face of a 128-channel readout system. Right: A similar image of a cosmic ray muon passing nearly coplanar to a fully-instrumented 832-channel readout system. Both figures display the raw data generated by the readout system; no filtering or signal enhancement has been applied.

Figure 9 shows the average self-triggered rate of each channel taken with the 28-channel system. The $50 \mathrm{mV}$ spread in the pedestal voltages among the channels exceeded the range over which we could tune relative differences in threshold among the channels. The self-triggering of the system was optimized by disabling a few percent of channels with the highest pedestal voltages (visible as gaps in figure 9), and the thresholds of the remaining channels were tuned to achieve sensitivity to MIP signals. After tuning, the majority of channels had self-trigger rates of roughly $0.02 \mathrm{~Hz}$, consistent with the estimated rate from cosmic ray muons. A few remaining channels with high pedestal voltages were kept active and gave manageable $0.05 \mathrm{~Hz}$ to $1 \mathrm{~Hz}$ trigger rates from noise, as shown in figure 9.

A 512-channel readout system was operated in a larger LArTPC system located at the University of Bern. The design of the LArTPC was similar, also $10 \mathrm{~cm}$ in diameter, but with a drift length of $60 \mathrm{~cm}$. In addition to the larger active volume, this LArTPC had substantially higher purity and 


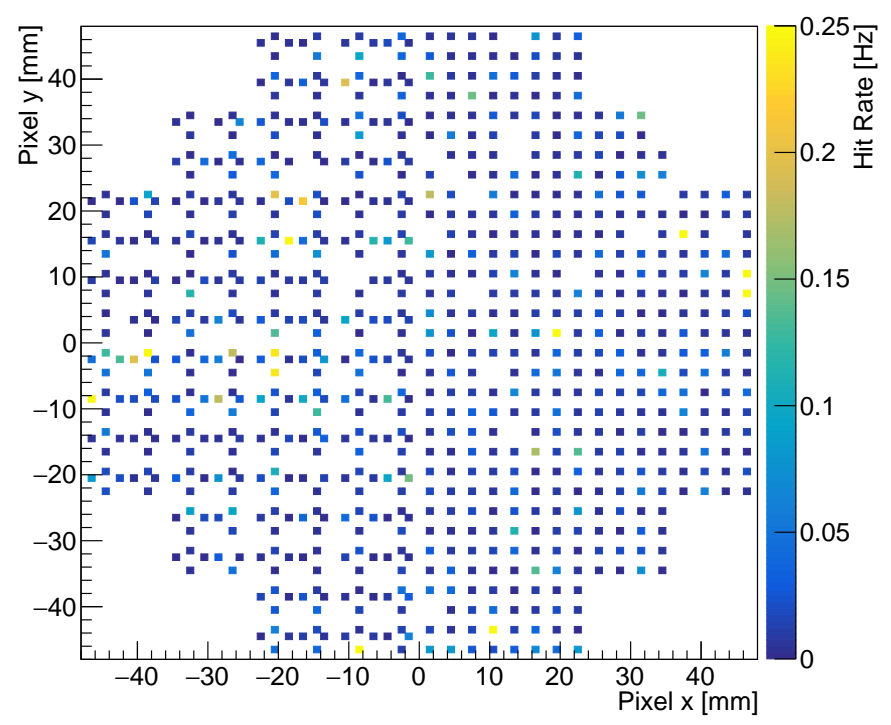

Figure 9. Channel self-trigger rates for an 832-channel pixelated readout system with $3 \mathrm{~mm}$ spacing. The majority of channels have low rates $(\sim 0.02 \mathrm{~Hz})$ that are dominated by the cosmic ray signals of interest. A few channels have low thresholds and high rates $(>0.05 \mathrm{~Hz})$ that are primarily noise. A few-percent of channels were disabled due to high noise rates, and are visible as gaps in the pixelated array.

therefore less electron loss. Liquid argon purity was maintained by occasional recirculation of the LAr through a purification system, enabling continuous operation over multiple days. The LArTPC was capable of operation at drift fields up to $1 \mathrm{kV} / \mathrm{cm}$, resulting in fewer electrons lost to prompt recombination. A description of this LArTPC system is given in [17]. The 512-channel readout system operated stably in this LArTPC for one week, with roughly half of the data collected at a drift field of $500 \mathrm{~V} / \mathrm{cm}$ and the other half at $1 \mathrm{kV} / \mathrm{cm}$. No degradation of readout performance was observed over this period. Figure 10 displays a few 3D images demonstrating the typical signals of cosmic ray interactions observed during this test. These images show raw data collected from the system; aside from conversion of ADC value to voltage, no filtering, enhancement, or other manipulation was applied. The higher purity of the argon and increased drift field, relative to the 10-cm-drift LArTPC, produced the more distinct 3D images shown in figure 10. See Supplemental Material at [26] for a 3D animation of cosmic ray events.

During operation of these readout systems, a low rate $(\sim 1 \mathrm{~Hz})$ of high-amplitude pulses was observed on a fraction of channels using the analog monitor of the ASICs. This pulsing was found to originate from bubbling of the LAr, driven by heat from the readout system located at the top face of the LArTPC. At LBNL we subsequently rotated the entire LArTPC by 90 degrees, so that the readout anode was now vertically-oriented and the electron drift was horizontal. The higher LAr head pressure and potentially improved convective cooling for this orientation was sufficient to completely suppress bubbling for both the 128-channel and 832-channel systems. The 512-channel readout system was located at the top face of the 60-cm LArTPC at Bern, but it was possible to adjust the level of the LAr in this cryostat. Adjusting the LAr level so that the readout system was immersed at a depth of at least $\sim 6 \mathrm{~cm}$ was sufficient to suppress bubbling in this system. 


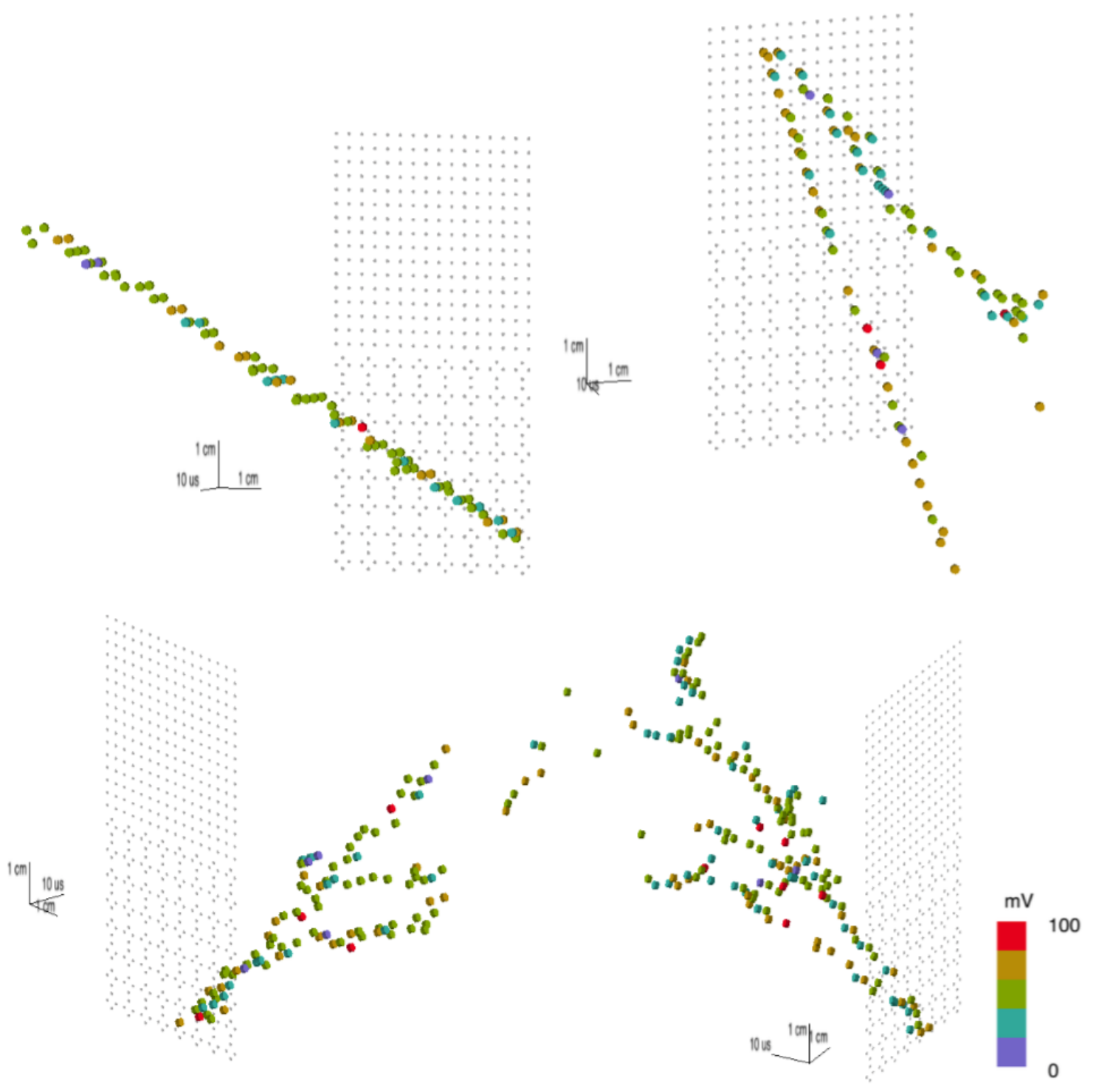

Figure 10. Four example 3D images of cosmic ray interactions detected using a $4.8 \mathrm{~cm}$ by $9.6 \mathrm{~cm}$ readout system in a 60-cm-drift LArTPC. The upper figures show relatively simple straight muon tracks, while the lower figures show small electromagnetic showers. This is raw data collected from the system; no filtering or enhancement has been applied.

During LArTPC testing we would occasionally collect data for the specific purpose of monitoring the stability of the CSA output pedestal voltage. This was achieved by briefly enabling each channel with a discriminator threshold set to the minimum value $(0 \mathrm{~V})$. The channel under test would self-trigger and digitize at the maximum allowed rate, $\sim 500 \mathrm{kHz}$ given the $5 \mathrm{MHz}$ clock, and transmit ADC values corresponding to zero input charge at the maximum $10 \mathrm{kHz}$ rate provided by the daisy chain communication. Based on these tests, the CSA pedestal voltages were found to be stable to within a few millivolts over the week of operation in the Bern LArTPC system. These data also served as a valuable test of the response to the system to bursts of high rate operation 
and consequent higher heating, far in excess of that expected from any reasonable LArTPC signals. No bubbling was observed under these extreme conditions, except for when the LAr level was very close to the $6 \mathrm{~cm}$ threshold.

\section{Discussion}

We have presented the design and results from operation of a low-power readout system which provides true 3D imaging of particle interactions in LArTPCs. This readout system substantially bested the design targets for noise $\left(500 \mathrm{e}^{-} \mathrm{ENC}\right)$ and power $(<100 \mu \mathrm{W} / \mathrm{channel})$, and also met the requirements for cryogenic operation $(87 \mathrm{~K})$ and digital multiplexing ( $>1000$ pads per data $\mathrm{I} / \mathrm{O}$ channel). The excellent performance of this first-generation system enabled instrumentation and operation of LArTPCs with drift lengths of $10 \mathrm{~cm}$ and $60 \mathrm{~cm}$. Signals from cosmic ray muons were imaged in 3D with no evidence of spurious hits from noise, and required no data filtering or signal enhancement. The improved fidelity of true 3D readout overcomes issues of signal ambiguities present for projective readout methods, which is one of the factors currently hindering reconstruction of neutrino-nuclear interactions at energies of a few $\mathrm{GeV}$. It should also enable LArTPC operation in a high-occupancy environments, such as the DUNE Near Detector site.

Using pixelated 3D readout for LArTPCs has other potential advantages. The rigid pixel PCBs are more mechanically robust and less sensitive to microphonic noise. Production of this readout relied on standard commercial electronics fabrication techniques, and so production of large detector readout can benefit from industrial economies of scale and QA/QC processes. Assembly of large-scale readout from small $\left(<0.25 \mathrm{~m}^{2}\right)$ modular tiles should ease readout testing and installation relative to large $\left(>10 \mathrm{~m}^{2}\right)$ wire-based readout planes. Neither ASIC production costs $\left(\sim \$ 2500 / \mathrm{m}^{2}\right)$ nor PCB fabrication costs $\left(\sim 500 / \mathrm{m}^{2}\right)$ are prohibitive for instrumentation of large detectors, although detailed methods for low-cost assembly and testing of large-scale readout systems are still in development. This readout may also have potential for large directional dark matter detectors that rely on negative ion drift for high-resolution imaging of nuclear recoils [27].

The next steps for 3D readout development begin with a detailed assessment of performance, including: gain stability, dynamic response, cross-talk, and longevity, among other aspects. It is also important to examine potential shortcomings of the current design via a program of measurements in combination with simulation. The use of a discriminator for signal timing adds slewing and other signal shape dependence to the estimation of the signal time. Simulation suggests this effect is generally small compared to the current $200 \mathrm{~ns}$ timestamp precision, and much smaller than the $2.2 \mu$ s resample time, but a detailed assessment is still required. The brief deadtime after each self-trigger will result in some charge loss for signals of long duration, such as tracks with a steep angle of incidence to the readout plane; a measurement of this effect using cosmic ray data is in progress. The impact of the loss of charge signals that are below the channel threshold must be similarly examined. The current daisy chain communication is not robust; the failure of one ASIC renders the chain broken. Revision of the current LArPix design would address some of the shortcomings described above, allow for enhanced features, and help address concerns about long-term cryogenic operation. Design and testing of a larger modular readout panel for tiling large surfaces would provide a natural step towards instrumenting large LArTPCs. 


\section{Acknowledgments}

We would like to thank our collaborators from the Laboratory for High Energy Physics at the Univ. of Bern (Antonio Ereditato, James Sinclair, Igor Kreslo, Damian Goeldi, among others) for their pioneering work in pixel readout of LArTPCs, for extensive discussions on readout design, for providing the 10-cm-drift LArTPC used for testing at LBNL, and for operation of the 60-cm-drift LArTPC at Bern. Dan McKinsey very kindly provided the high-purity liquid argon system for hosting this LArTPC at LBNL, and Lucie Tvrznikova went out of her way to help us adapt and operate this system. The consistently useful suggestions from Yuan Mei at LBNL helped us avoid a variety of pitfalls during both design and testing of these readout systems. This material is based upon work supported by the U.S. Department of Energy, Office of Science, Office of High Energy Physics, under contract number DE-AC02-05CH11231.

\section{References}

[1] J. Marx and D. Nygren, The Time Projection Chamber, Phys. Today 31 (1978) 46.

[2] C. Patrignani et al, Review of Particle Physics, Chin. Phys. C40 (2016) 100001.

[3] C. Rubbia, The Liquid Argon Time Projection Chamber: A New Concept for Neutrino Detectors, CERN-EP-INT-77-08 (1977).

[4] H. H. Chen, P. E. Condon, B. C. Barish, and F. J. Sciulli, A Neutrino detector sensitive to rare processes. I. A Study of neutrino electron reactions, FERMILAB-PROPOSAL-0496 (1976).

[5] W. J. Willis and V. Radeka, Liquid Argon Ionization Chambers as Total Absorption Detectors, Nucl. Instrum. Meth. 120 (1974) 221.

[6] S. Amerio et al, Design, construction and tests of the ICARUS T600 detector, Nucl. Instrum. Meth. A527 (2004) 329.

[7] C. Anderson et al, The ArgoNeuT Detector in the NuMI Low-Energy beam line at Fermilab, JINST 7 (2012) 10019.

[8] R. Acciarri et al, Design and Construction of the MicroBooNE Detector, JINST 12 (2017) 02017.

[9] M. Antonello et al, A Proposal for a Three Detector Short-Baseline Neutrino Oscillation Program in the Fermilab Booster Neutrino Beam, arXiv:1503.01520.

[10] R. Acciarri et al, Long-Baseline Neutrino Facility (LBNF) and Deep Underground Neutrino Experiment (DUNE), arXiv:1601.05471.

[11] R. Acciarri et al, Noise Characterization and Filtering in the MicroBooNE Liquid Argon TPC, JINST 12 (2017) 08003.

[12] C. Adams et al, Ionization Electron Signal Processing in Single Phase LArTPCs I. Algorithm Description and Quantitative Evaluation with MicroBooNE Simulation, JINST 13 (2018) 07006.

[13] C. Adams et al, Ionization Electron Signal Processing in Single Phase LArTPCs II. Data/Simulation Comparison and Performance in MicroBooNE, JINST 13 (2018) 07007.

[14] X. Qian, C. Zhang, B. Viren, and M. Diwan, Three-dimensional Imaging for Large LArTPCs, JINST 13 (2018) 05032.

[15] J. Alme et al, The ALICE TPC, a large 3-dimensional tracking device with fast readout for ultra-high multiplicity events, Nucl. Instrum. Meth. A622 (2010) 316. 
[16] M. Anderson et al, The Star time projection chamber: A Unique tool for studying high multiplicity events at RHIC, Nucl. Instrum. Meth. A499 (2003) 659.

[17] J. Asaadi et al, First Demonstration of a Pixelated Charge Readout for Single-Phase Liquid Argon Time Projection Chambers, arXiv:1801.08884.

[18] M. Miyajima, T. Takahashi, S. Konno, T. Hamada, S. Kubota, H. Shibamura, and T. Doke, Average energy expended per ion pair in liquid argon, Phys. Rev. A9 (1974) 1438.

[19] M. Miyajima, T. Takahashi, S. Konno, T. Hamada, S. Kubota, H. Shibamura, and T. Doke, Erratum: Average energy expended per ion pair in liquid argon, Phys. Rev. A10 (1974) 1452.

[20] S. Amoruso et al, Study of electron recombination in liquid argon with the ICARUS TPC, Nucl. Instrum. Meth. A523 (2004) 275.

[21] R. Acciarri et al, A study of electron recombination using highly ionizing particles in the ArgoNeuT Liquid Argon TPC, JINST 8 (2013) 08005.

[22] M. Antonello et al, Experimental observation of an extremely high electron lifetime with the ICARUS-T600 LAr-TPC, JINST 9 (2014) 12006.

[23] R. Acciarri et al, First Observation of Low Energy Electron Neutrinos in a Liquid Argon Time Projection Chamber, Phys. Rev. D95 (2017) 072005.

[24] W. Walkowiak, Drift velocity of free electrons in liquid argon, Nucl. Instrum. Meth. A449 (2000) 288.

[25] L. Tvrznikova, Ph.D Thesis (in preparation), Yale University 2018.

[26] http://arxiv.org/src/1808.02969/anc/larpix_cosmics_60cmTPC.mp4.

[27] J. B. R. Battat et al, Readout technologies for directional WIMP Dark Matter detection, Phys. Rept. $662(2016) 1$. 